\title{
Identification of Extra Virgin Olive Oils Modified by the Addition of Soybean Oil, Using Ion Chromatography
}

\author{
Patrícia T. Souza, ${ }^{a}$ Marina Ansolin, ${ }^{b}$ Eduardo A. C. Batista, ${ }^{b}$ Antonio J. A. Meirelles ${ }^{b}$ \\ and Matthieu Tubino ${ }^{\circledR *, a}$ \\ ${ }^{a}$ Instituto de Química, Universidade Estadual de Campinas (UNICAMP), P.O. Box 6154, \\ 13083-970 Campinas-SP, Brazil \\ ${ }^{b}$ Faculdade de Engenharia de Alimentos, Universidade Estadual de Campinas (UNICAMP), \\ 13083862 Campinas-SP, Brazil
}

\begin{abstract}
The modification of four extra virgin olive oils from different origins with refined soybean oil (SO), in the proportions of $0,5,10,20$ and $30 \% \mathrm{~m} / \mathrm{m}$, was studied using two approaches. First, the induction period (IP) was determined by Rancimat. In the second strategy, ion chromatography was used to quantify the formic and acetic acids, in their carboxylate forms (formate and acetate), present in the water contained in the measurement vessel of the Rancimat device, $21 \mathrm{~h}$ after the beginning of the induced oxidation of the samples. The content of the adulterant $(\mathrm{SO})$ was linearly correlated with the IP (mean correlation coefficient $(\mathrm{R})=0.9858$ ) and the concentration of the formate (mean $\mathrm{R}=0.9979$ ) and acetate (mean $\mathrm{R}=0.9951$ ). Also, it was observed that as the IP decreased and the formic acid and acetic acid concentrations increased, the monounsaturated fatty acids/polyunsaturated fatty acids ratio decreased.
\end{abstract}

Keywords: extra virgin olive oil, soybean oil, modification, short chain carboxylic acids, oxidative stability

\section{Introduction}

Extra virgin olive oils (EVOOs) are obtained from olive fruits by cold pressing and other mechanical or physical processes, under conditions that do not change the natural features of olive oil. The EVOO acidity, expressed as

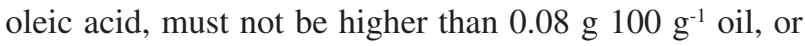
$0.2 \mathrm{mg} \mathrm{KOH} \mathrm{g}^{-1}$ oil. $^{1}$

The importance of controlling the olive oil quality is due to the increase in its worldwide consumption and its nutritional and sensory properties. The International Olive Council (IOC) $)^{2}$ data for 2017 and prediction for 2018 indicate the European Union as the primary producer and exporter. Spain, Italy, Greece and Portugal are the largest world producers and exporters. In the import sector, the United States takes the lead, followed by the European Union and Brazil.

For the quality control and determination of olive oils adulterated with lower-priced vegetable oils, chemometrics has been combined with analytical techniques, such as

*e-mail: tubino@iqm.unicamp.br ultraviolet-visible spectroscopy, infrared spectroscopy, nuclear magnetic resonance spectroscopy and other strategies, such as liquid chromatography and gas chromatography. ${ }^{3-8}$

Lipid oxidation is the reaction of oxygen with unsaturated fatty chains and is the main cause of lipid deterioration. The unsaturation level (number of double bonds) of vegetable oils, depends on their fatty acid composition. ${ }^{9}$ For example, the main fatty acid of soybean oil (SO) is linoleic acid (C18:2), which contains two double bonds, ${ }^{10}$ whereas the main fatty acid of olive oil is oleic acid (C18:1), which comprises only one double bond. ${ }^{1}$

Allylic hydrogen atoms are susceptible to oxidation reactions, leading to the formation of hydroperoxides, whereas the oxidative reactivity increases for bis-allylic hydrogen atoms present in polyunsaturated fatty acid (poly FA) chains. ${ }^{9}$ These degradation processes are not proportional to the number of double bonds in the molecule but to the number of bis-allylic sites. ${ }^{11}$

The oxidative stability of olive oils is associated with its natural antioxidants, such as phenolic compounds, tocopherols, carotenoids and chlorophylls. ${ }^{12-16}$ 
The oil oxidative stability is determined through the oil stability index, also known as the induction period (IP), according to the official method (Cd 12b-92) of the American Oil Chemists' Society (AOCS), ${ }^{17}$ using the Rancimat equipment. This method induces the acceleration of the sample oxidation by its exposure to controlled air flow and high temperature. In the Rancimat procedure, the volatile compounds formed during oxidation are collected in deionized water contained in the equipment measurement vessel, thereby increasing the water conductivity, which is monitored. Previously, the oxidative stability (IP) of different kinds of olive oils measured by Rancimat presented a linear correlation with the oleic/linoleic acid ratio and with the phenolic compounds and tocopherol content. ${ }^{12}$

Recently, formic and acetic acids were determined in the water of the Rancimat measurement vessel during the induced oxidation monitoring of soybean oil, and methyl and ethyl biodiesels from soybean oil. ${ }^{18}$ The same approach was applied to biodiesel from soybean oil ${ }^{19}$ and biodiesel from rapeseed oil. ${ }^{20}$ In the induced oxidation equipment, known as the active oxygen method, ${ }^{21}$ formic and acetic acids were identified as being the major components among the volatile acids determined during the degradation monitoring of soybean, peanut, rapeseed, corn, sunflower and olive oils..$^{22}$ The analytical methods that have been employed to determine formic and acetic acids in lipid samples include capillary electrophoresis ${ }^{23}$ and ion chromatography. ${ }^{18-20,24-26}$

In the present work, two methods were studied to determine the modification of EVOO with refined soybean oil. The first one was based on the IP, determined by Rancimat. In the second approach, ion chromatography was used for quantifying the formic and acetic acids as carboxylates (formate and acetate) contained in the water of the Rancimat measurement vessel, after $21 \mathrm{~h}$ from the beginning of the induced oxidation of the samples. These two methods were chosen to be studied because they have simple experimental execution and relatively low cost.

\section{Experimental}

\section{Materials, reagents and equipment}

Four EVOOs from different origins (EVOO-1, EVOO-2, EVOO-3 and EVOO-4) and refined soybean oil were bought from the local market. The samples were stored at room temperature before being analyzed.

Sodium formate (99\%) and lithium chloride (analytical grade) were from Quimis (São Paulo, Brazil). Sodium acetate (99\%), ethyl acetate (analytical grade), methanol (analytical grade) and sulfuric acid (analytical grade) were purchased from Synth (São Paulo, Brazil). Acetone and hexane (both high-performance liquid chromatography (HPLC) grade) were supplied by Tedia (California, CA, USA). 2,2-Diphenyl-1-picrylhydrazyl (DPPH; $>99 \%$ ), 1,10-phenanthroline (> 99\%; Phen) and ferrous sulfate $(99 \%)$ were acquired from Sigma-Aldrich (St. Louis, MO, USA). Deionized water (resistivity 18.2 $\mathrm{M} \Omega \mathrm{cm}$ ) was obtained by using a Milli-Q system from Millipore (Darmstadt, Germany). The hydrophilic polytetrafluoroethylene (PTFE) $0.45 \mu \mathrm{m}$ filter was also from Millipore.

The Rancimat (model 837), ion chromatograph (model 882 Compact) equipped with a Metrosep Organic Acids $250 / 7.8(250 \times 7.8 \mathrm{~mm})$ column and automatic sampler (model 863) were all from Metrohm (Herisau, Switzerland). The gas chromatograph with flame ionization detector (model Clarus 600) was from PerkinElmer (Massachusetts, MA, USA), and the UV-Vis spectrophotometer (Ultrospec 2000) was from Pharmacia Biotech (Cambridge, England).

\section{Soybean oil and EVOO characterization}

The fatty acid composition of the samples was determined by gas chromatography, according to the AOCS Ce1-62 official method. ${ }^{17}$ Before chromatographic analysis, refined soybean oil and the EVOOs were transesterified to fatty acid methyl esters, according to Hartman and Lago. ${ }^{27}$

The acidity value (AOCS Cd 3d-63) and water content (AOCS Ca 2e-84) of the samples were measured based on official methods. ${ }^{17}$

The tocopherol content in the samples was established, in triplicate, according to Ansolin et al..$^{28}$

The total oil fraction, without previous extraction, and the methanolic fraction of the samples were measured through the DPPH radical scavenging method. The antioxidant activity of the methanolic fraction was also determined through the Phen method. Both, the methanol extracts and the Phen method were performed according to Szydłowska-Czerniak et al. ${ }^{29}$ The reaction conditions of the DPPH method were based on Espin et al..$^{30}$ The DPPH antioxidant activity (in percentage) was calculated as $\left[\left(\mathrm{Abs}_{\text {control }}-\mathrm{Abs}_{\text {sample }}\right) / \mathrm{Abs}_{\text {control }}\right] \times 100$, where $\mathrm{Abs}$ is the absorbance after $30 \mathrm{~min}$ of reaction. The antioxidant activity by the Phen method was calculated from an analytical curve, using $\mathrm{FeSO}_{4} \cdot 7 \mathrm{H}_{2} \mathrm{O}$ as the standard.

\section{Modification of the EVOO samples}

The EVOO samples were modified with soybean oil, which was added in the proportions of $0,5,10,20$ and 
$30 \% \mathrm{~m} / \mathrm{m}$ to form $30.00 \pm 0.01 \mathrm{~g}$ of the blend. The blends were homogenized by vortexing for $1 \mathrm{~min}$. The IP of each of these samples was determined in triplicate by the Rancimat procedure according to the AOCS Cd 12b-92 official method. ${ }^{17}$

\section{Quantification of formic and acetic acids (as formate and acetate)}

Formic and acetic acids were determined in their anionic form as carboxylates (formate and acetate), in the water contained in the Rancimat measurement vessel, $21 \mathrm{~h}$ after the beginning of induced oxidation. The water contained in the Rancimat measurement vessel, which received the acids transported from the reaction vessel, was filtered using a $0.45 \mu \mathrm{m}$ hydrophilic filter. Then, it was injected into the ion chromatograph. As described by Souza et al., ${ }^{18}$ the chromatographic conditions were as follows: Metrosep
Organic Acids column (Metrohm); eluent, 80:20 v/v water:acetone with $0.5 \mathrm{mmol} \mathrm{L}^{-1}$ sulfuric acid; suppressor solution, $20 \mathrm{mmol} \mathrm{L}{ }^{-1} \mathrm{LiCl}$; flow rate, $0.4 \mathrm{~mL} \mathrm{~min}^{-1}$; injection volume, $20 \mu \mathrm{L}$; running time, $1 \mathrm{~h}$. The analytical curves were constructed from 10 points in the concentration range of 1 to $1000 \mathrm{mg} \mathrm{L}^{-1}$ for each carboxylate (formate and acetate). Each point of the curve was determined in triplicate, i.e., three injections of each concentration were done. Each analytical curve was always reconstructed at the beginning of the analysis, for each sample series. The samples were injected in triplicate, in all cases.

\section{Results and Discussion}

\section{Soybean oil and EVOO characterization}

Table 1 reports the characterization of the soybean oil and EVOOs, before the induced oxidation by Rancimat.

Table 1. Characterization of the soybean oil (SO) and of the extra virgin olive oils (EVOOs) from different origins

\begin{tabular}{|c|c|c|c|c|c|c|}
\hline \multirow[b]{2}{*}{ Fatty acid } & \multirow[b]{2}{*}{ Symbol $^{\mathrm{b}}$} & \multicolumn{5}{|c|}{ Sample $^{\mathrm{a}}$} \\
\hline & & $\begin{array}{c}\mathrm{SO} / \\
(\% \mathrm{~m} / \mathrm{m})\end{array}$ & $\begin{array}{l}\text { EVOO-1/ } \\
(\% \mathrm{~m} / \mathrm{m})\end{array}$ & $\begin{array}{l}\text { EVOO-2 / } \\
(\% \mathrm{~m} / \mathrm{m})\end{array}$ & $\begin{array}{l}\text { EVOO-3/ } \\
(\% \mathrm{~m} / \mathrm{m})\end{array}$ & $\begin{array}{l}\text { EVOO-4 / } \\
(\% \mathrm{~m} / \mathrm{m})\end{array}$ \\
\hline Palmitic & $\mathrm{C} 16: 0$ & $11.01 \pm 0.01$ & $11.00 \pm 0.03$ & $14.29 \pm 0.01$ & $11.23 \pm 0.04$ & $12.61 \pm 0.01$ \\
\hline Palmitoleic & C16:1 & $0.09 \pm 0.01$ & $0.74 \pm 0.01$ & $1.42 \pm 0.01$ & $0.84 \pm 0.01$ & $0.89 \pm 0.03$ \\
\hline Margaric & $\mathrm{C} 17: 0$ & $0.07 \pm 0.01$ & $0.08 \pm 0.02$ & $0.05 \pm 0.02$ & $0.07 \pm 0.02$ & $0.06 \pm 0.01$ \\
\hline Heptadecenoic & $\mathrm{C} 17: 1$ & $0.05 \pm 0.01$ & $0.14 \pm 0.01$ & $0.09 \pm 0.01$ & $0.10 \pm 0.01$ & $0.13 \pm 0.05$ \\
\hline Stearic & $\mathrm{C} 18: 0$ & $3.25 \pm 0.03$ & $2.99 \pm 0.01$ & $2.79 \pm 0.02$ & $3.06 \pm 0.03$ & $2.35 \pm 0.03$ \\
\hline Oleic & $\mathrm{C} 18: 1$ & $23.28 \pm 0.01$ & $78.25 \pm 0.01$ & $69.59 \pm 0.04$ & $78.47 \pm 0.01$ & $75.47 \pm 0.01$ \\
\hline Linoleic & $\mathrm{C} 18: 2$ & $54.58 \pm 0.02$ & $5.42 \pm 0.02$ & $10.45 \pm 0.02$ & $4.88 \pm 0.02$ & $7.04 \pm 0.02$ \\
\hline trans-Linoleic & $\mathrm{C} 18: 2 t^{\mathrm{c}}$ & $0.11 \pm 0.01$ & - & - & - & - \\
\hline Linolenic & $\mathrm{C} 18: 3$ & $6.66 \pm 0.01$ & $0.72 \pm 0.03$ & $0.65 \pm 0.01$ & $0.74 \pm 0.02$ & $0.70 \pm 0.01$ \\
\hline Arachidic & $\mathrm{C} 20: 0$ & $0.32 \pm 0.01$ & $0.33 \pm 0.01$ & $0.36 \pm 0.08$ & $0.33 \pm 0.01$ & $0.35 \pm 0.02$ \\
\hline Gadoleic & $\mathrm{C} 20: 1$ & $0.20 \pm 0.01$ & $0.25 \pm 0.01$ & $0.22 \pm 0.01$ & $0.21 \pm 0.01$ & $0.28 \pm 0.01$ \\
\hline Behenic & $\mathrm{C} 22: 0$ & $0.38 \pm 0.01$ & $0.09 \pm 0.01$ & $0.09 \pm 0.01$ & $0.08 \pm 0.01$ & $0.11 \pm 0.01$ \\
\hline Monounsaturated & & $23.62 \pm 0.01$ & $79.38 \pm 0.01$ & $71.32 \pm 0.02$ & $79.62 \pm 0.01$ & $76.77 \pm 0.03$ \\
\hline Polyunsaturated & & $61.35 \pm 0.02$ & $6.14 \pm 0.03$ & $11.10 \pm 0.02$ & $5.62 \pm 0.02$ & $7.74 \pm 0.02$ \\
\hline Acidity value / (mg KOH g $\left.{ }^{-1}\right)$ & & $0.04 \pm 0.01$ & $0.02 \pm 0.01$ & $0.02 \pm 0.02$ & $0.02 \pm 0.01$ & $0.03 \pm 0.02$ \\
\hline Water content / $\left(\mathrm{mg} \mathrm{kg}^{-1}\right)$ & & $523 \pm 6$ & $511 \pm 3$ & $741 \pm 1$ & $528 \pm 5$ & $625 \pm 5$ \\
\hline$\alpha$-Tocopherol / (mg kg$\left.{ }^{-1}\right)$ & & $113 \pm 1$ & $207.1 \pm 0.3$ & $212 \pm 1$ & $218.85 \pm 0.06$ & $168 \pm 2$ \\
\hline$\gamma$-Tocopherol / $\left(\mathrm{mg} \mathrm{kg}^{-1}\right)$ & & $1171.7 \pm 0.9$ & $24.07 \pm 0.07$ & $16.2 \pm 0.6$ & $24.65 \pm 0.07$ & $8.7 \pm 0.4$ \\
\hline$\delta$-Tocopherol / $\left(\mathrm{mg} \mathrm{kg}^{-1}\right)$ & & $240 \pm 2$ & - & - & - & - \\
\hline Total tocopherol / $\left(\mathrm{mg} \mathrm{kg}^{-1}\right)$ & & $1525 \pm 2$ & $231.2 \pm 0.3$ & $228 \pm 1$ & $243.50 \pm 0.07$ & $177 \pm 2$ \\
\hline Induction period / h & & $11.2 \pm 0.4$ & $24.1 \pm 0.3$ & $21.5 \pm 0.2$ & $26.0 \pm 0.8$ & $19.2 \pm 0.2$ \\
\hline \multirow{2}{*}{ DPPH antioxidant activity / \% } & total oil fraction & $93.8 \pm 0.6$ & $71.7 \pm 0.3$ & $68.8 \pm 0.1$ & $73.6 \pm 0.4$ & $59.8 \pm 0.5$ \\
\hline & methanolic fraction & $15.7 \pm 0.2$ & $12.9 \pm 0.3$ & $5.1 \pm 0.1$ & $14.4 \pm 0.3$ & $2.5 \pm 0.1$ \\
\hline $\begin{array}{l}\text { Phen method antioxidant } \\
\text { activity / }\left(\mu \mathrm{mol} \mathrm{Fe} \mathrm{Fe}^{\mathrm{II}} 100 \mathrm{~g}^{-1}\right)\end{array}$ & methanolic fraction & $325.2 \pm 0.4$ & $142.5 \pm 0.3$ & $125.8 \pm 0.2$ & $156.3 \pm 0.8$ & $72.9 \pm 0.2$ \\
\hline Storage time $\mathrm{d}^{\mathrm{d}}$ months & & 2.5 & 9.5 & 12 & 7.5 & 14 \\
\hline
\end{tabular}

${ }^{\mathrm{a} S O}$ : soybean oil, EVOO: extra virgin olive oil; ${ }^{\mathrm{b}} \mathrm{Cx}: \mathrm{y}$, x: number of carbon atoms, y: number of double bonds; ${ }^{\mathrm{c}}$ trans isomer; ${ }^{\mathrm{d}}$ storage time (in months) after the date of manufacture shown on the packaging that the samples were stored before being analyzed. DPPH: 2,2-diphenyl-1-picrylhydrazyl; Phen: 1,10-phenanthroline. 
As expected, the fatty acid composition of the EVOOs characteristically contained more oleic (C18:1) than linoleic acid (C18:2). Conversely, the soybean oil includes more linoleic (C18:2) than oleic acid (C18:1) and more linolenic acid (C18:3) than the EVOOs. The fatty acid compositions of the olive and the soybean oils reported in Table 1 are consistent with the compositions stated in the literature. ${ }^{1,10,31}$ However, as the composition $(\% \mathrm{~m} / \mathrm{m})$ described in the literature comprises a range for each fatty acid, there is a comfortable margin for adulteration of olive oil without significantly affecting the accepted fatty acid content.

The tocopherol content and the IP values related to the olive oils in Table 1 corroborated with the compositions reported in earlier work. ${ }^{12}$ Similarly, the tocopherols measured in the soybean oil are in agreement with the composition reported in the literature. ${ }^{31}$ In addition, the EVOOs presented an acidity lower than $0.2 \mathrm{mg} \mathrm{KOH} \mathrm{g}^{-1}$ oil, and the SO had an acidity less than $0.6 \mathrm{mg} \mathrm{KOH} \mathrm{g}^{-1}$ oil, as reported previously for these kinds of oils. ${ }^{1,10}$

The EVOO antioxidant activities and the total tocopherol content presented the same order as the IP measured by the Rancimat procedure where: EVOO-3 > EVOO-1 > EVOO-2 > EVOO-4 (Table 1). This behavior concurs with the results obtained by Aparicio et al., ${ }^{12}$ which showed that the IP was positively correlated with the content of antioxidants in the olive oils. Moreover, the total tocopherol content decreases during storage of the oils, ${ }^{32}$ as can be seen in Table 1 for the EVOO samples.

The IP of the soybean oil was around 50\% lower than those of the EVOOs. It is possible to correlate this result with the fact that soybean oil contains a higher concentration of poly FAs than EVOOs (Table 1). Aparicio et al..$^{12}$ reported that the oleic/linoleic acid ratio is more important for the oil stability than the total tocopherol content.

The antioxidant activity of the soybean oil, measured by Phen and DPPH methods, was higher than the antioxidant activity of EVOOs. Likewise, the total tocopherol content in the soybean oil was also higher than in the EVOO (Table 1). The commercial soybean oil used in this work had tert-butylhydroquinone in addition to its natural antioxidants. This synthetic antioxidant is widely used in vegetable oils because of its excellent antioxidant activity. ${ }^{16,33,34}$ Furthermore, the lipid oxidation mechanism shows significant alterations under high temperature and oxygen flow, with the occurrence of lateral reactions usually irrelevant at room temperature. As a consequence, the formation of new antioxidant or pro-oxidant species may occur, which can present contradictory results in measurements performed under high temperature. . $^{35,36}$

When there are high tocopherol concentrations in oils, these antioxidants can be consumed by side reactions, as in the hydroperoxides decomposition. This behavior can decrease the oxidative stability of the oils. ${ }^{37,38}$

\section{Determination of the EVOO modification based on the IP}

Figure 1 shows that the IP is almost linearly correlated with the soybean oil content, added as the adulterant in EVOO samples. Table 2 provides the linear regression data.

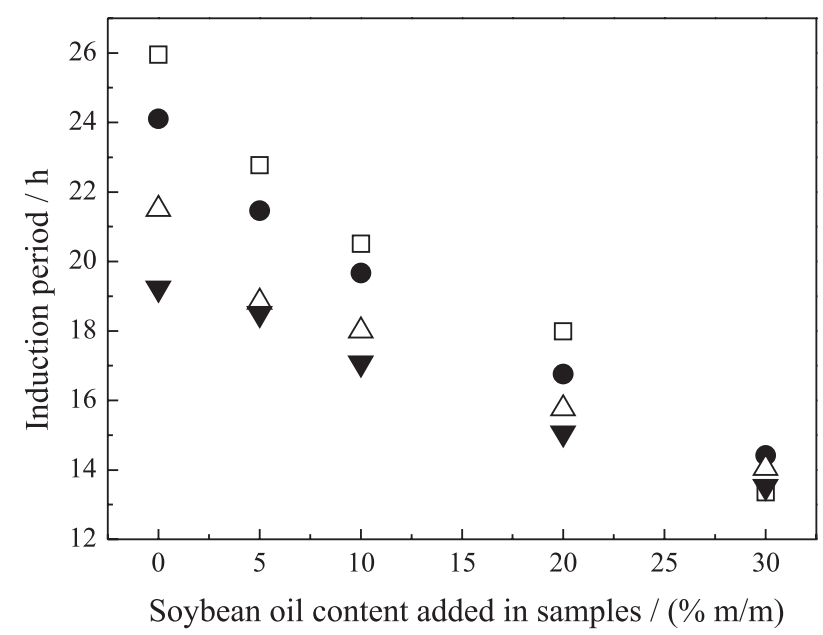

Figure 1. Correlations between induction period (IP) and soybean oil (SO) content added as an adulterant in the extra virgin olive oil (EVOO) samples. The IP of the SO (100\%) is $11.2 \pm 0.4 \mathrm{~h}$. Q: EVOO- $1+\mathrm{SO}$; $\triangle$ : EVOO-2 + SO; $\square$ : EVOO-3 + SO; $\mathbf{\nabla}:$ EVOO-4 + SO.

Table 2. Correlation coefficient $(\mathrm{R})$ and the linear regression equations which correlate the induction period (IP) versus the percentage of the soybean oil (SO) content (in $\% \mathrm{~m} / \mathrm{m}$ ), added as an adulterant in the extra virgin olive oils (EVOOs) studied

\begin{tabular}{lcc}
\hline Sample & $\begin{array}{c}\text { Correlation } \\
\text { coefficient }(\mathrm{R})\end{array}$ & IP equation $/ \mathrm{h}$ \\
\hline EVOO-1 + SO & 0.9842 & $\mathrm{IP}=23.2-0.31 \mathrm{SO} \%$ \\
EVOO-2 + SO & 0.9909 & $\mathrm{IP}=20.0-0.20 \mathrm{SO} \%$ \\
EVOO-3 + SO & 0.9758 & $\mathrm{IP}=25.0-0.35 \mathrm{SO} \%$ \\
EVOO-4 + SO & 0.9921 & $\mathrm{IP}=19.2-0.20 \mathrm{SO} \%$ \\
\hline
\end{tabular}

${ }^{\mathrm{a} S O} \%$ : content of soybean oil (in $\% \mathrm{~m} / \mathrm{m}$ ) in the extra virgin olive oil.

Figure 1 suggests that an EVOO with IP lower than $23 \mathrm{~h}$ may have been adulterated or stored for a long time, and therefore it contains low levels of antioxidants, with a consequent reduction of its oxidative stability. In this case, the EVOO should be analyzed more carefully with respect to its quality.

In the accelerated oxidation process of lipid samples at high temperature and continuous air flow, according to the Rancimat procedure, the order of unsaturated chains reactivity is: linolenic $(\mathrm{C} 18: 3)>$ linoleic $(\mathrm{C} 18: 2)>>$ oleic (C18:1). ${ }^{9,18,20,39,40}$ The fatty acid composition was found to be the most decisive factor in the oxidation of EVOO with 
palm oil blends by the Rancimat procedure, in which case the increase of the IP showed a linear correlation with the increasing palm oil content in the blends. This trend was correlated with the increase of the saturated fatty acids/ unsaturated fatty acids ratio. ${ }^{41}$

In another study on the oxidative stability of various kinds of olive oils, the monounsaturated fatty acids/ polyunsaturated fatty acids ratio was considered as an appropriate measure of the olive oil tendency to undergo autoxidation, with higher levels of this ratio meaning higher olive oil oxidative stability ${ }^{42}$ In this context, the IP can be related to the oleic (C18:1)/linoleic (C18:2) acid ratio in the samples (Figure 2a) and also with the monounsaturated fatty acids/polyunsaturated fatty acids ratio (Figure $2 b$ ). For the oleic (C18:1)/linoleic (C18:2) acid ratio, the linoleic and trans-linoleic chains were considered as C18:2. For the mono FA/poly FA ratio, the following mono FA chains were considered: palmitoleic acid (C16:1), heptadecenoic acid (C17:1), oleic acid (C18:1) and gadoleic acid (C20:1). The polyunsaturated chains were linoleic acid (C18:2), trans-linoleic acid (C18:2) and linolenic acid (C18:3). These ratios were calculated, considering the composition presented in Table 1 and the mass percentages $(\% \mathrm{~m} / \mathrm{m})$ used in the modification of the EVOO with soybean oil.

It is possible to see in Figure 2a that with the decrease of the oleic (C18:1)/linoleic (C18:2) ratio, the IP value diminishes. Similarly, with the decline in the monounsaturated fatty acids/polyunsaturated fatty acids ratio, the IP also decreased (Figure $2 b$ ).

\section{Determination of EVOO modification based on the formic} and acetic acids

The increase of the conductivity of water contained in the measurement vessel, during the induced oxidation Rancimat procedure, results from the contribution of formic, acetic and other acids formed in the lipid oxidation process. The variation of the conductivity with time describes the curve used to determine the IP of the sample..$^{18,20}$

The samples were maintained under induced oxidation by Rancimat for the fixed time of $21 \mathrm{~h}$, after which, the water contained in the Rancimat measurement vessel, where the conductivity is continuously measured, was analyzed by ion chromatography. The fixed time of $21 \mathrm{~h}$ was chosen, due to comparative purposes according to the data in Figure 1, where the majority of the samples showed an IP lower than $21 \mathrm{~h}$. Therefore, during this time, the collected water for analysis is expected to contain a significant concentration of formic and acetic acids formed in the induced oxidation. Some samples that showed an IP higher than $21 \mathrm{~h}$ have been analyzed in the intermediate
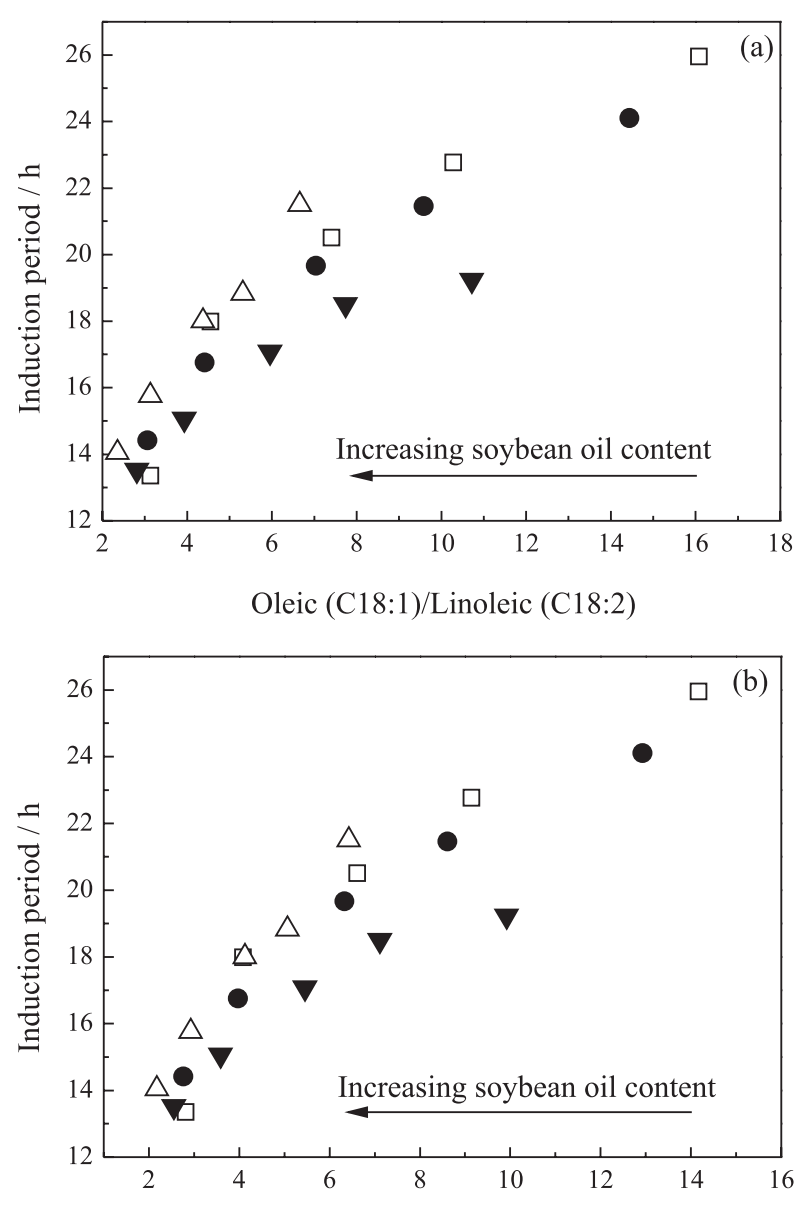

Monounsaturated fatty acids / Polyunsaturated fatty acids

Figure 2. (a) Correlation between induction period and oleic (C18:1)/ linoleic (C18:2) acid ratio content in the samples; (b) correlation between induction period and monounsaturated fatty acids/polyunsaturated fatty acids ratio content in the samples. O: EVOO- $1+\mathrm{SO} ; \triangle$ : EVOO- $2+\mathrm{SO}$; $\square$ : EVOO-3 + SO; $\mathbf{\nabla}$ : EVOO-4 + SO (SO: soybean oil; EVOO: extra virgin olive oil).

stage of the induced oxidation (region with insignificant formic and acetic acid concentrations but close to the IP). The formic and acetic acids were quantified by their carboxylates (formate and acetate).

The formate (Figure 3a) and acetate (Figure 3b) concentrations were higher in the samples with higher levels of adulterant content (SO), when compared to the pure EVOOs. The curves in Figures $3 \mathrm{a}$ and $3 \mathrm{~b}$ indicated that EVOO-1 + SO and EVOO- 3 + SO samples presented higher oxidative stability than EVOO-2 + SO and EVOO-4 + SO samples, and displayed a polynomial behavior. Instead, EVOO-2 + SO and EVOO-4 + SO curves exhibited a linear response (Figures $3 \mathrm{a}$ and $3 \mathrm{~b}$ ). It can be explained because the samples EVOO- $1+0 \%$ SO, EVOO- $1+5 \%$ SO, EVOO-3 + 0\% SO and EVOO-3 + 5\% SO had an IP longer than $21 \mathrm{~h}$ and had been analyzed in the intermediate stage of the induced oxidation (region with insignificant formic and acetic acid concentration but close to the IP). 
Considering only the linear ranges of Figures $3 \mathrm{a}$ and $3 \mathrm{~b}$, the linear regression equation corresponding to each sample was determined (Table 3).

The formate and acetate concentrations were higher for EVOO-2 + SO and EVOO-4 + SO samples than for

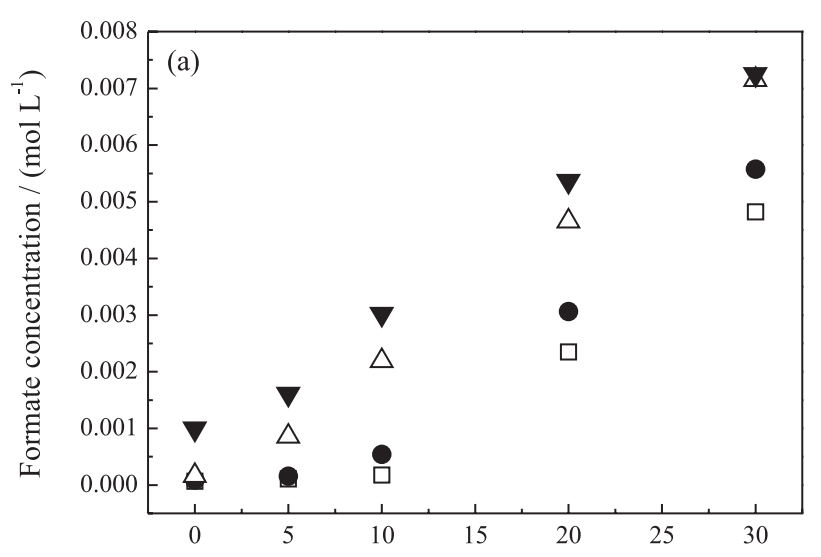

Soybean oil content added in sample / $(\% \mathrm{~m} / \mathrm{m})$

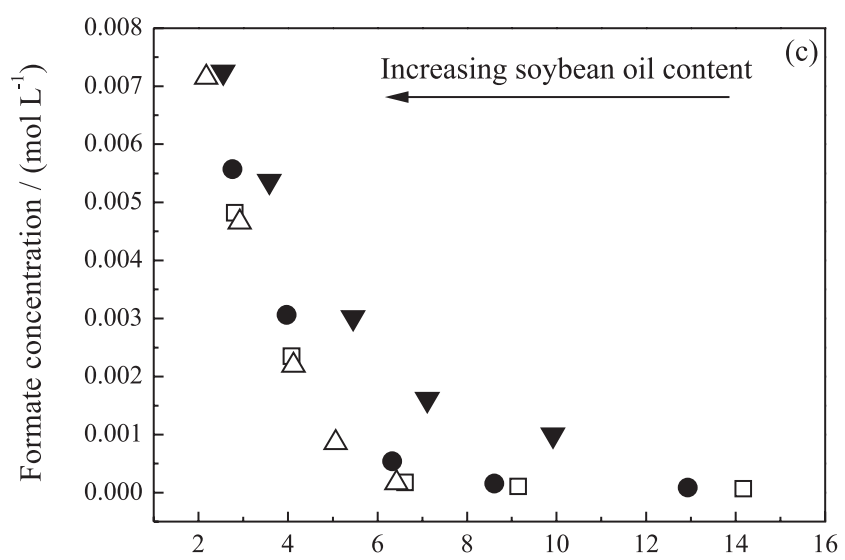

Monounsaturated fatty acids / Polyunsaturated fatty acids
EVOO-1 + SO and EVOO-3 + SO samples (Figures 3a and $3 \mathrm{~b})$. This behavior is in accordance with the fact that the EVOO-2 + SO and EVOO-4 + SO samples have lower oxidative stability than EVOO-1 + SO and EVOO-3 + SO samples (Figure 1). Also, the formate concentration was
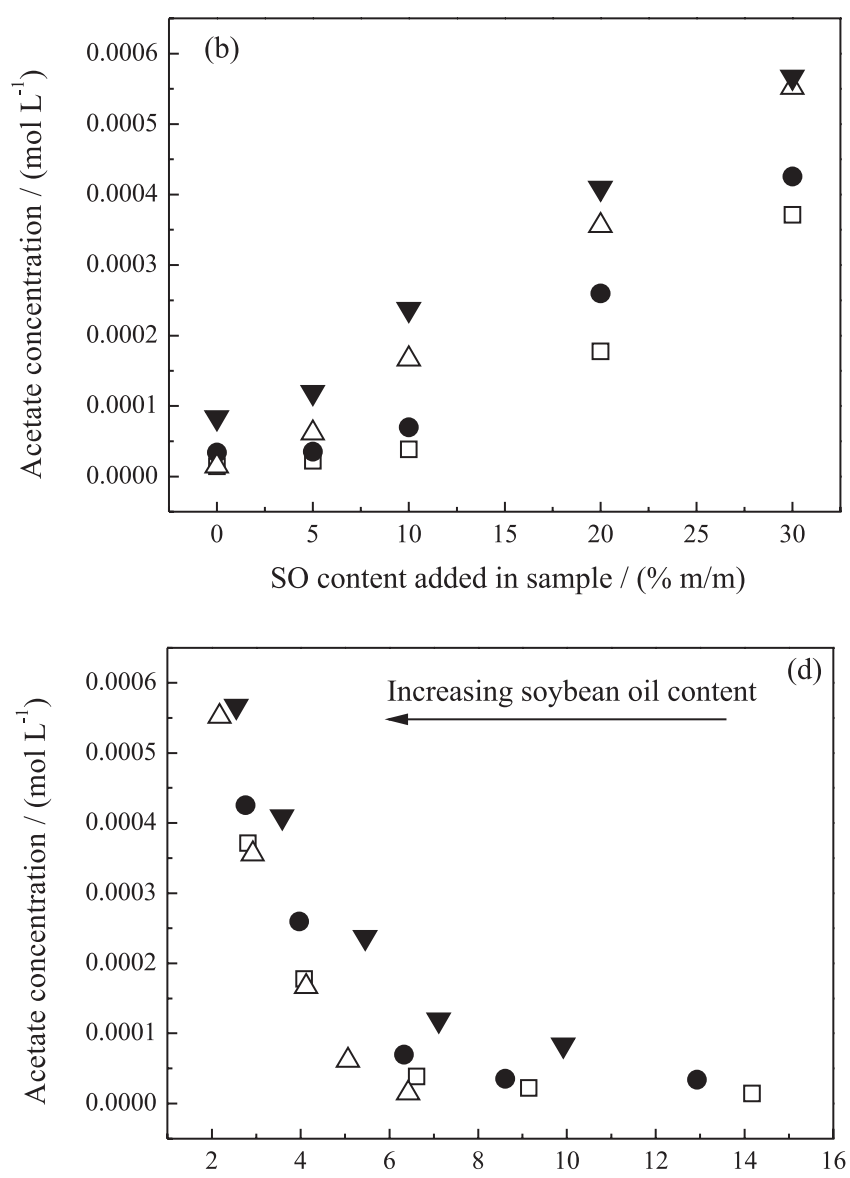

Monounsaturated fatty acids / Polyunsaturated fatty acids

Figure 3. (a) Correlation between formate concentration and the soybean oil adulterant content; (b) correlation between acetate concentration and the soybean oil adulterant content; (c) correlation between formate concentration and the monounsaturated fatty acids/polyunsaturated fatty acids ratio; (d) correlation between acetate concentration and the monounsaturated fatty acids/polyunsaturated fatty acids ratio. $\bullet:$ EVOO- $1+\mathrm{SO} ; \triangle$ : EVOO-2 + SO; $\square$ : EVOO-3 + SO; $\mathbf{\nabla}:$ EVOO-4 + SO (EVOO: extra virgin olive oil; SO: soybean oil).

Table 3. Linear regression equations of the modification curves correlating the formic and acetic acids concentrations (quantified from their carboxylates formate $\left(\mathrm{C}_{\text {Formate }}\right)$ and acetate $\left(\mathrm{C}_{\text {Acetate }}\right)$, respectively) with the content of the adulterant (soybean oil; $\left.\mathrm{SO}\right)$ in the extra virgin olive oils (EVOOs)

\begin{tabular}{llccl}
\hline Anion & Sample $^{\mathrm{a}}$ & Linear range / $\%$ adulterant $)$ & Correlation coefficient $(\mathrm{R})$ & \multicolumn{1}{c}{ Equation $/(\mathrm{mol} \mathrm{L})^{-1}$} \\
\hline \multirow{3}{*}{ Formate } & EVOO-1 + SO & $10-30$ & 0.9999 & $\mathrm{C}_{\text {Formate }}=-0.0020+0.000252 \mathrm{SO} \%$ \\
& EVOO-2 + SO & $0-30$ & 0.9971 & $\mathrm{C}_{\text {Formate }}=-0.0001+0.000239 \mathrm{SO} \%$ \\
& EVOO-3 + SO & $10-30$ & 0.9986 & $\mathrm{C}_{\text {Formate }}=-0.0022+0.000232 \mathrm{SO} \%$ \\
& EVOO-4 + SO & $0-30$ & 0.9959 & $\mathrm{C}_{\text {Formate }}=0.0008+0.000217 \mathrm{SO} \%$ \\
\hline \multirow{3}{*}{ Acetate } & EVOO-1 + SO & $10-30$ & 0.9985 & $\mathrm{C}_{\text {Acetate }}=-0.00010+0.0000178 \mathrm{SO} \%$ \\
& EVOO-2 + SO & $0-30$ & 0.9960 & $\mathrm{C}_{\text {Acetate }}=-0.00001+0.0000184 \mathrm{SO} \%$ \\
& EVOO-3 + SO & $10-30$ & 0.9912 & $\mathrm{C}_{\text {Acetate }}=-0.00014+0.0000166 \mathrm{SO} \%$ \\
& EVOO-4 + SO & $0-30$ & 0.9947 & $\mathrm{C}_{\text {Acetate }}=0.00006+0.0000168 \mathrm{SO} \%$ \\
\hline
\end{tabular}

${ }^{\text {a SO: }}$ soybean oil, EVOO: extra virgin olive oil; ${ }^{\mathrm{b}} \mathrm{SO} \%=$ content of soybean oil $(\% \mathrm{~m} / \mathrm{m})$ in the extra virgin olive oil. 
about 10 times higher than the acetate concentration, for all the samples analyzed. This result is consistent with the literature data, which reports that the formic acid is the preponderant short chain carboxylic acid, among the other products of the lipid oxidation. . $^{18-20,22-26}$

The adulterant ( $\mathrm{SO}$ ) content changes the fatty acid composition of the blend (EVOO + SO). Therefore, it is possible to state that the concentrations of formic and acetic acids, produced in the lipid oxidation, are influenced by the fatty acid composition of the sample. The data obtained suggest that an EVOO with a formate concentration higher than $5 \times 10^{-4} \mathrm{~mol} \mathrm{~L}^{-1}$ (Figure 3a) or acetate concentration higher than $5 \times 10^{-5} \mathrm{~mol} \mathrm{~L}^{-1}$ (Figure $3 \mathrm{~b}$ ), after submission to the same conditions under study, may have been adulterated.

Figures $3 \mathrm{c}$ and $3 \mathrm{~d}$ show that the formate and acetate concentrations increase following the decrease of the monounsaturated fatty acids/polyunsaturated fatty acids ratio. Based on this observation, it is possible to infer that the formic and acetic acids are products of the unsaturated fatty chains oxidation, and they are mainly produced from the polyunsaturated fatty acids chains.

Considering Figures $3 \mathrm{c}$ and $3 \mathrm{~d}$, it can be observed that when the monounsaturated fatty acids/polyunsaturated fatty acids ratio is lower than eight, there is an accentuated increase in the formate (Figure 3c) and in the acetate concentrations (Figure 3d). Thus, it is possible to suppose that an EVOO sample with a monounsaturated fatty acids/ polyunsaturated fatty acids ratio lower than eight may have been adulterated and should be analyzed more carefully.

Comparison between the methods proposed in this work and others existent in the literature

The proposed method for the identification of EVOO modified by the addition of soybean oil based on IP does not require preparation of samples and use of reagents and solvents other than water. Although the waiting time for the result to be approximately $24 \mathrm{~h}$, the interpretation of the data is easy and done directly on the computer screen without requiring statistical treatment.

The second method proposed for the identification of EVOO modified by the addition of soybean oil is based on the determination of formic and acetic acids formed along the time. For the preparation of the samples is used the Rancimat procedure, which is simple to perform and in which it is not necessary the use of reagents or solvents other than water. Formic and acetic acids are analyzed through ion chromatography, which is a relatively simple method to perform ${ }^{18}$ and which uses small quantities of simple and low cost reagents such as sodium acetate, sodium formate, lithium chloride, acetone and water, all presenting low environmental impact.

In addition, the results obtained by the two methods proposed in this work directly reflect the overall composition of the samples, including the fatty and the antioxidants compositions. They also reflect the storage time, not being restricted to a signal of a single chemical group as in the case of other existing procedures that use infrared spectroscopy ${ }^{4-6}$ and nuclear magnetic resonance spectroscopy, ${ }^{5}$ which are based on signals related only to triacylglycerols. Other procedures using liquid chromatography ${ }^{3,8}$ or gas chromatography $y^{7,8}$ are also based on signals related to groups of molecules (triacylglycerols, phenolic compounds, sterols). They additionally require previous preparation steps of the samples including extraction, separation and derivatization of the compounds of interest, which make use of toxic reagents and solvents.

It must be also remembered that most of the existing methods in the literature ${ }^{3-8}$ make use of chemometrics to treat the data, while the data offered by the two methods proposed in this paper are given by direct interpretation of the experimental results.

\section{Conclusions}

The IP of each sample presented a linear correlation with the amount of soybean oil used as an adulterant in the EVOO samples. Furthermore, with the decrease of the monounsaturated fatty acids/polyunsaturated fatty acids ratio of these samples, the IP was decreased. This behavior can be assigned to the polyunsaturated fatty acids chains, which present a higher reactivity towards oxygen than polyunsaturated fatty acids chains, and is reflected in the decrease of the oxidative stability of the mixture with soybean oil relative to the pure EVOO.

The formate and acetate concentrations, determined at $21 \mathrm{~h}$ after the beginning of the induced oxidation by Rancimat, showed a linear behavior with respect to the soybean oil content added to the EVOO. Furthermore, as the monounsaturated fatty acids/polyunsaturated fatty acids ratio in these samples decreased, the formate and acetate concentrations increased. These results confirm that the formic and acetic acids are oxidation products formed mainly by the polyunsaturated fatty acids chains.

The data obtained suggest that an EVOO sample with a monounsaturated fatty acids/polyunsaturated fatty acids ratio lower than eight, IP less than $23 \mathrm{~h}$ or (after submission to the same conditions under study) a formate concentration above $5 \times 10^{-4} \mathrm{~mol} \mathrm{~L}^{-1}$ or acetate concentration above $5 \times 10^{-5} \mathrm{~mol} \mathrm{~L}^{-1}$, may have been adulterated. Therefore, in this case, the EVOO should be analyzed more carefully. 
The methods studied showed adequate correlations to indicate the modification of extra virgin olive oils by the addition of soybean oil. Therefore, they fulfilled the objectives of this work. In addition, they have simple experimental execution and relatively low cost.

\section{Acknowledgments}

This work was supported by FAPESP (project Nos. 2014/21252-0 and 2017/12015-3) and CNPq (project Nos. 404808/2013-1, 406856/2013-3, 420868/2016-0, 406963/2016-9 and 308924/2017-7).

\section{References}

1. Codex Alimentarius; Standard for Olive Oils and Olive Pomace Oils, CX-STAN 33-1981, Revision 2 (2003), Amendment 2 (2013); FAO/WHO: Rome, Italy, 1981.

2. http://www.internationaloliveoil.org/estaticos/view/131-worldolive-oil-figures/, accessed in September 2018.

3. Bajoub, A.; Medina-Rodríguez, S.; Gómez-Romero, M.; Ajal, E. A.; Bagur-González, M. G.; Fernández-Gutiérrez, A.; CarrascoPancorbo, A.; Food Chem. 2017, 215, 245.

4. Georgouli, K.; Del Rincon, J. M.; Koidis, A.; Food Chem. 2007, 17,735 .

5. Gómez-Caravaca, A. M.; Maggio, R. M.; Cerretani, L.; Anal. Chim. Acta 2016, 913, 1.

6. de Luca, M.; Restuccia, D.; Clodoveo, M. L.; Puoci, F.; Ragno, G.; Food Chem. 2016, 202, 432.

7. Yang, Y.; Ferro, M. D.; Cavaco, I.; Liang, Y.; J. Agric. Food Chem. 2013, 61, 3693.

8. Jabeur, H.; Zribi, A.; Makni, J.; Rebai, A.; Abdelhedi, R.; Bouaziz, M.; J. Agric. Food Chem. 2014, 62, 4893.

9. Frankel, E. N.; Lipid Oxidation, $2^{\text {nd }}$ ed.; The Oily Press: Bridgwater, UK, 2005.

10. Codex Alimentarius; Standard for Named Vegetables Oils, CX-STAN 210-1999, Revision 3 (2009), Amendment 3 (2013); FAO/WHO: Rome, Italy, 1999.

11. Knothe, G.; Fuel Process. Technol. 2007, 88, 669.

12. Aparicio, R.; Roda, L.; Albi, M. A.; Gutiérrez, F.; J. Agric. Food Chem. 1999, 47, 4150.

13. Borges, T. H.; Pereira, J. A.; Cabrera-Vique, C.; Lara, L.; Oliveira, A. F.; Seiquer, I.; Food Chem. 2017, 215, 454.

14. Franco, M. N.; Galeano-Díaz, T.; López, O.; FernándezBolaños, J. G.; Sánchez, J.; de Miguel, C.; Gil, M. V.; MartínVertedor, D.; Food Chem. 2014, 163, 289.

15. Evans, J. C.; Kodali, D. R.; Addis, P. B.; J. Am. Oil Chem. Soc. 2002, 79, 47.

16. Sherwin, E. R.; J. Am. Oil Chem. Soc. 1976, 53, 430.

17. American Oil Chemists' Society (AOCS); Official Methods and Recommended Practices of the AOCS, $6^{\text {th }}$ ed.; AOCS Press: Champaign, IL, 2009.

18. Souza, P. T.; Ansolin, M.; Batista, E. A. C.; Meirelles, A. J. A.; Tubino, M.; Fuel 2017, 199, 239.

19. de Carvalho, A. L.; Cardoso, E. A.; da Rocha, G. O.; Teixeira, L. S. G.; Pepe, I. M.; Grosjean, D. M.; Fuel 2016, 173, 29.

20. Flitsch, S.; Neu, P. M.; Schober, S.; Kienzl, N.; Ullmann, J.; Mittelbach, M.; Energy Fuels 2014, 28, 5849.

21. deMan, J. M.; deMan, L.; J. Am. Oil Chem. Soc. 1984, 61, 534.

22. deMan, J. M.; Tie, F.; deMan, L.; J. Am. Oil Chem. Soc. 1987, 64, 993.

23. Nogueira, T.; do Lago, C. L.; Microchem. J. 2011, 99, 267.

24. Silveira, E. L. C.; de Caland, L. B.; Tubino, M.; Fuel 2014, 124, 97.

25. Strömberg, N.; Sahlin, E.; Fuel 2012, 97, 531.

26. Zhang, Y.; Thepsithar, P.; Jiang, X.; Tay, J. H.; Energy Fuels 2014, 28, 2581.

27. Hartman, L.; Lago, R. C. A.; Lab. Pract. 1973, 22, 475.

28. Ansolin, M.; Souza, P. T.; Meirelles, A. J. A.; Batista, E. A. C.; Food Anal. Methods 2017, 10, 2165.

29. Szydłowska-Czerniak, A.; Dianoczki, C.; Recseg, K.; Karlovits, G.; Szłyk, E.; Talanta 2008, 76, 899.

30. Espin, J. C.; Soler-Rivas, C.; Wichers, H. J.; J. Agric. Food Chem. 2000, 48, 648.

31. Firestone, D.; Physical and Chemical Characteristics of Oils, Fats, and Waxes, $2^{\text {nd }}$ ed.; AOCS Press: Washington, USA, 2006.

32. Abramovič, H.; Butinar, B.; Nikolič, V.; Food Chem. 2007, 104, 903.

33. Ramalho, V. C.; Jorge, N.; Quim. Nova 2006, 29, 755.

34. Wanasundara, P. K. J. P. D.; Shahidi, F.; Bailey's Industrial Oil and Fat Products, vol. 1; Shahidi, F., ed.; John Wiley \& Sons, Inc.: New Jersey, USA, 2005, ch. 11.

35. Kamal-Eldin, A.; Yanishlieva N.; Analysis of Lipid Oxidation; Kamal-Eldin, A.; Pokorny, J., eds.; AOCS Publishing: Champaign, IL, 2005.

36. Silva, F. A. M.; Borges, M. F. M.; Ferreira, M. A.; Quim. Nova 1999, 22, 94.

37. Satue, M. T.; Huang, S. W.; Frankel, E. N.; J. Am. Oil Chem. Soc. 1995, 72, 1131.

38. Yanishlieva, N. V.; Kamal-Eldin, A.; Marinova, E. M.; Toneva, A. G.; Eur. J. Lipid Sci. Technol. 2002, 104, 262.

39. Cosgrove, J. P.; Church, D. F.; Pryor, W. A.; Lipids 1987, 22, 299.

40. Holman, R. T.; Elmer, O. C.; J. Am. Oil Chem. Soc. 1947, 24, 127.

41. de Leonardis, A.; Macciola, V.; Food Chem. 2012, 135, 1769.

42. Farhoosh, R.; Hoseini-Yazdi, S. Z.; Food Chem. 2013, 141, 557.

Submitted: September 19, 2018

Published online: January 11, 2019 